\title{
Implementation of factitious force method for control of 5R manipulator with skid-steering platform REX
}

\author{
A. MAZUR* and M. CHOLEWIŃSKI \\ Chair of Cybernetics and Robotics, Faculty of Electronics, \\ Wroclaw University of Science and Technology, 11/17 Janiszewskiego St., 50-372 Wroclaw, Poland
}

\begin{abstract}
In the paper implementation of the factitious force concept for a controlling complex mobile manipulator has been presented. As the nonholonomic constraint only lack of longitudinal slippage of wheels has been chosen - in skid-steering platforms lateral slippage is necessary to change orientation of such a platform. From a control theory point of view such a system is dynamically underactuated. As a solution to a underactuation problem a method of factitious force has been proposed. This method assumes extension on the dynamics level, in the form of an additional control inputs $u_{v}$, which values are equal to zero equivalently. For a mobile manipulator, consisting of platform REX and 5R robotic onboard arm, a cascaded control law has been proposed. A simulation study was conducted for a mathematical model of a considered object with real values of physical parameters, i.e. lengths, masses, inertia moments etc. obtained from the 3D model. Results obtained in simulations have shown a proper action of the control system and convergence of tracking errors, occurring in a platform and in joints of a manipulator, to zero.
\end{abstract}

Key words: underactuated system, nonholonomic constraint, kinematics, dynamics.

\section{Introduction}

A mobile manipulator, which is the subject of considerations, consists of a mobile platform and an onboard rigid manipulating arm. Such a robotic object can execute more complicated tasks than its components. A manipulating arm is fully controlled while a mobile platform equipped with more than one axis of fixed wheels is a dynamically underactuated system.

Wheeled mobile platforms can be treated as independent robots or as a transportation part of the complex robotic assemble, for instance mobile manipulators [1]. Depending on the wheels' type and a way in which they are fixed to the chassis, motion of wheeled mobile platforms can be realized with or without slipping effect. If no slippage effect between wheels and surface occurs, then there exists an equation describing forbidden directions for realized velocities of the system. Such an equation is called a nonholonomic constraint in platform's motion.

A special kind of wheeled mobile platforms are platforms with tracks. They can be modeled as a chassis equipped with more than one axis of fixed wheels. These platforms are called skid-steering mobile platforms, due to skidding effect observed in theirs behavior. An example of such a platform is the platform REX developed under the grant RobREx, see Fig. 1.

Designing control algorithms for skid-steering platforms is a challenging task. First attempts to solve this problem consisted of adding some artificial assumption about lateral slip during the platform's motion. Such an assumption can be considered as an artificial nonholonomic constraint [2] reducing a number of admissible control inputs in an object. The similar approach can be found e.g. in [3] or [4].

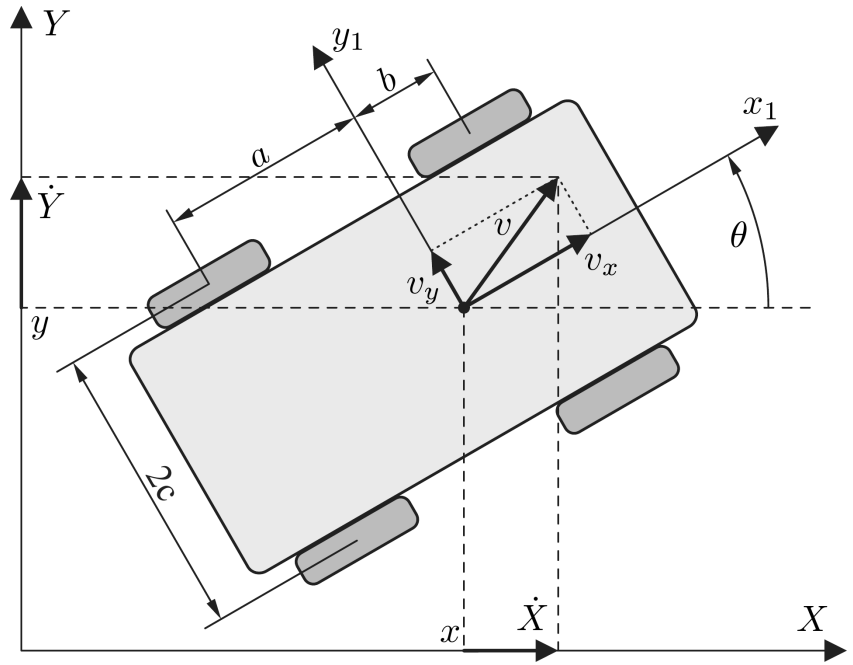

Fig. 1. Differentially driven skid-steering mobile platform REX

Another approach to the control problem for skid-steering platform has been presented in [5]. Authors have shown that the skid-steering platform is an underactuated system on a dynamic level with non-stationary kinematics (non-stationary velocity constraint). They have used the tunable dynamic oscillator to get globally uniformly bounded stability of the proposed control algorithm. The same idea can be found in [6] and [7].

First announcements about a new concept in control of the skid-steering platform can be found in [8]. The main idea of proposed method was to add a control input, so-called "factitious force", to make input matrix $B^{*}$ square invertible matrix.

*e-mail: alicja.mazur@pwr.edu.pl 
Because this additional control input does not exist in reality, it was assumed that it is equal to zero.

The main advantage of factitious force is the fact, that it makes possible to predict slipping effect and include them into a model of the moving platform. It is similar to the idea of "slipping variables" [9] which have really improved quality of realized trajectories of a platform moving with wheels' slippage.

In this paper a new approach to the control problem of mobile manipulator's with the skid-steering platform has been presented. As it has been mentioned earlier, a mobile manipulator with such a platform can be considered as the dynamically underactuated system. Till now every control algorithm presented in literature tried to avoid a problem of missing control inputs, e.g. assuming additional constraints, because the underactuated mobile platform has got rectangular input matrix (which is non-invertible). A problem with inverting such a matrix (standing before control signals) can be solved with the idea of factitious force. This is the essential novelty in control of skid-steering mobile vehicles.

The paper is organized in a following way. Section 2 illustrates theoretical design of the mathematical model of the nonholonomic mobile platform REX. In Sec. 3 a concept of the factitious force approach to the control nonholonomic mobile manipulators is presented. A model of the whole mobile manipulator including a nonholonomic platform and holonomic $5 \mathrm{R}$ manipulator is presented in Sec. 4. In Sec. 5 the control problem is formulated. In Sec. 6 the main result, i.e. new control algorithm including kinematic and dynamic control law, is designed. Section 7 contains the simulation results. Section 8 presents some conclusions.

\section{Mathematical model of platform REX}

Object description. In the paper the skid-steering mobile platform REX, presented in Fig. 1, is considered. Each pair of wheels on the same side is coupled by the transmission belt or the track, thus the angular velocities of wheels on the same side are the same. Additionally, it means that the resulting torque acting on one side of a platform is a sum of torques generated from both motors.

The distances from the front and back axis of robot wheels to the center of the mass are denoted as $b$ and $a$ respectively. The half of the platform width is signed as $c$.

The state vector of a platform can be denoted as follows

$$
q_{m}=\left(\begin{array}{lllll}
x & y & \theta & \phi_{1} & \phi_{2}
\end{array}\right)^{T} \in R^{5},
$$

where $x, y$ are cartesian coordinates of the platform's mass center, $\theta$ is orientation of the platform and $\phi_{1}$ and $\phi_{2}$ are the rotational angles of wheels at left and right side, respectively.

Dynamics of mobile platform can be derived from the Lagrange formula

$$
L_{p}\left(q_{m}, \dot{q}_{m}\right)=E_{k p}-E_{p p}
$$

The platform moves on an equipotential plane and therefore, its potential energy is equal to zero $\left(E_{p p}=0\right)$, hence, the Lagrange formula is composed only of kinetic energy of a platform and its wheels

$$
\begin{aligned}
L_{p}= & \frac{1}{2}\left(I_{z}+4 I_{z z}+m_{k} \sum_{i=1}^{4} d_{i}^{2}\right) \dot{\theta}^{2}+\frac{1}{2} I_{x x}\left(\dot{\phi}_{1}^{2}+\dot{\phi}_{2}^{2}\right) \\
& +\frac{1}{2}\left(m_{p}+4 m_{k}\right)\left(\dot{x}^{2}+\dot{y}^{2}\right)=\frac{1}{2} \dot{q}_{m}^{T} M_{p} \dot{q}_{m},
\end{aligned}
$$

where $m_{p}, m_{k}$ are mass of a platform and a wheel respectively. In turn, $I_{z}$ is inertia moment of a platform and $I_{z z}$ and $I_{x x}$ are inertia moments of wheel (expressed relative to local $Z$ and $X$ axis). The distance between platform's center of mass and the point of contact of $i$-th wheel with the ground is marked as $d_{i}$. The symbol $M_{p}$ denotes inertia matrix for platform REX

$$
M_{p}=\left[\begin{array}{ccccc}
m_{t} & 0 & 0 & 0 & 0 \\
0 & m_{t} & 0 & 0 & 0 \\
0 & 0 & I_{p} & 0 & 0 \\
0 & 0 & 0 & I_{x x} & 0 \\
0 & 0 & 0 & 0 & I_{x x}
\end{array}\right]
$$

with elements defined below

- $I_{p}=I_{z}+4 I_{z z}+m_{k} \sum_{i=1}^{4} d_{i}^{2}-$ total inertia moment of platform with wheels,

- $m_{t}=m_{p}+4 m_{k}$ - total mass of a platform,

- $I_{x x}=\frac{1}{2} m_{k} r^{2}-$ inertia moment of wheel relative to rotation axis.

Kinematics. In the skid-steering platforms the lateral slippage is needed to change the orientation. Thus, only the constraints for lack of longitudinal slippage can be introduced to the system equations.

Nonholonomic constraints coming from lack of longitudinal slippage can be expressed in the following Pfaffian form [3]

$$
A\left(q_{m}\right) \dot{q}_{m}=\left[\begin{array}{ccccc}
\cos \theta & \sin \theta & -c & -r & 0 \\
\cos \theta & \sin \theta & c & 0 & -r
\end{array}\right]\left(\begin{array}{c}
\dot{x} \\
\dot{y} \\
\dot{\theta} \\
\dot{\phi}_{1} \\
\dot{\phi}_{2}
\end{array}\right)=0
$$

where $r$ is a radius of platform wheel.

2.1. Model in generalized coordinates. Dynamics of a mobile platform can be derived from the d'Alembert principle [10] and have the following form

$$
M\left(q_{m}\right) \ddot{q}_{m}+F\left(q_{m}, \dot{q}_{m}\right)=B\left(q_{m}\right) u_{m}+A^{T}\left(q_{m}\right) \lambda,
$$

where $M\left(q_{m}\right)=M_{p}$ is constant inertia matrix of platform given by (3), $B\left(q_{m}\right)$ is input matrix describing which state variables are directly actuated by motors, $u_{m}$ is a vector of control inputs, $A^{T}\left(q_{m}\right) \lambda$ describes forces of nonholonomic constraints and $\lambda$ is a vector of Lagrange multipliers. In turn, $F\left(q_{m}, \dot{q}_{m}\right)$ is vector of non-conservative forces, responsible for friction and reaction forces of a ground, described as below 


$$
F\left(q_{m}, \dot{q}_{m}\right)=\left[\begin{array}{c}
F_{x} \cos \theta-F_{y} \sin \theta \\
F_{x} \sin \theta+F_{y} \cos \theta \\
M_{r} \\
0 \\
0
\end{array}\right],
$$

where [2]

$$
\begin{gathered}
F_{x}=\sum_{i=1}^{4} F_{x i}, \quad F_{x i}=\mu \operatorname{sgn}\left\{\dot{x}_{i}\right\} \\
F_{y}=\sum_{i=1}^{4} F_{y i}, \quad F_{y i}=f \operatorname{sgn}\left\{\dot{y}_{i}\right\} \\
M_{r}=a\left(F_{y 1}+F_{y 2}\right)-b\left(F_{y 3}+F_{y 4}\right) \\
+c\left(F_{x 1}+F_{x 3}-F_{x 2}-F_{x 4}\right) .
\end{gathered}
$$

$F_{x i}$ is $i$-th wheel static force, which turn agrees with direction of a wheel, and $F_{y i}$ is static friction force, perpendicular to the vehicle motion. Coefficients $\mu$ and $f$ are the static friction coefficients along, respectively, $i$-th wheel local $X_{i}$ and $Y_{i}$ axis. $M_{r}$ is resultant moment, responsible for platform orientation. A detailed description of reaction forces can be found in [2] or [3].

As it was mentioned earlier, the skid-steering platform is provided with side torques generated by the sum of all motors torques at a given side. Hence, the input matrix has the following form

$$
B\left(q_{m}\right)=\left[\begin{array}{lllll}
0 & 0 & 0 & 0 & 1 \\
0 & 0 & 0 & 1 & 0
\end{array}\right]^{T} .
$$

Control theory of noholonomic objects stands, that if the state vector has $n$ elements and there is $l$ nonholonomic constraints introduced to the system, the set of control inputs must be equal to $m=n-l[11]$.

2.2. Model in auxiliary velocities. Since due to (4) the platform velocity $\dot{q}_{m}$ is in a null space of matrix $A\left(q_{m}\right)$, it is always possible [12] to find a vector of special auxiliary velocities $\eta \in R^{m}$, such that

$$
\dot{q}_{m}=G\left(q_{m}\right) \eta
$$

where $G$ is an $n \times m$ full rank matrix satisfying the relation$\operatorname{ship} A\left(q_{m}\right) G\left(q_{m}\right)=0$.

Dynamics. After substituting the Eq. (9) into the dynamics (5) we get

$$
M^{*} \dot{\eta}+C^{*} \eta+F^{*}=B^{*} u_{m}
$$

with elements defined in the following way

$$
\begin{array}{cc}
M^{*}=G^{T} M_{p} G, & C^{*}=G^{T} M_{p} \dot{G}, \\
F^{*}=G^{T} F, & B^{*}=G^{T} B .
\end{array}
$$

Equation (10) describes the dynamics of nonholonomic mobile platform expressed in the auxiliary coordinates.
Kinematics. For the platform REX size $n$ of the state vector $q_{m}$ is equal to 5 from (1) and number of nonholonomic constraints for lack of longitudinal slippage equals to $l=2$. It strictly means, that matrix $G\left(q_{m}\right)$ should have three independent columns spanning kernel of $A\left(q_{m}\right)$ matrix.

As it can be concluded from (9), real velocity of the platform $\dot{q}_{m}$ can be expressed as linear combination of auxiliary velocities $\eta$. It is very desirable if signals $\eta_{i}$ have physical meaning, therefore control inputs $\eta_{1}$ and $\eta_{2}$ are usually chosen as angular velocities of left and right wheels in differentially driven platform with one axis of fixed wheels. From this point of view two columns in $G\left(q_{m}\right)$ i.e. $G_{1}$ and $G_{2}$ are determined and only third column should be selected in appropriate way

$$
G\left(q_{m}\right)=\left[\begin{array}{cc|c}
\cos \theta & \cos \theta & a_{1} \\
\sin \theta & \sin \theta & a_{2} \\
\frac{1}{c} & -\frac{1}{c} & a_{3} \\
0 & \frac{2}{r} & a_{4} \\
\frac{2}{r} & 0 & a_{5}
\end{array}\right]=\left[\begin{array}{ll|l}
G_{1} & G_{2} & G_{3}
\end{array}\right] .
$$

Third column of matrix $G\left(q_{m}\right)$ is selected properly only if following conditions are fulfilled:

- First condition: column $G_{3}$ has to be linearly independent from $G_{1}$ and $G_{2}$, hence

$$
\operatorname{rank} G\left(q_{m}\right)=3 \text {. }
$$

- Second condition: column $G_{3}$ has to belong to kernel of $A\left(q_{m}\right)$ matrix, i.e. $A\left(q_{m}\right) G_{3}=0$. It means that following equations can be fulfilled

$$
\begin{aligned}
& a_{1} \cos \theta+a_{2} \sin \theta-a_{3} c-a_{4} r=0, \\
& a_{1} \cos \theta+a_{2} \sin \theta+a_{3} c-a_{5} r=0 .
\end{aligned}
$$

Subtracting sides of Eqs. (14) and (15) the following condition can be obtained

$$
a_{5}-a_{4}=\frac{2}{c} a_{3} .
$$

Input matrix. The most interesting part of dynamics (10) is the input matrix $B^{*}\left(q_{m}\right)$. For matrix $G$ selected as in (12) such matrix has a form

$$
B^{*}\left(q_{m}\right)=\left[\begin{array}{cc}
\frac{2}{r} & 0 \\
0 & \frac{2}{r} \\
a_{5} & a_{4}
\end{array}\right] .
$$

This matrix is rectangular and, consequently, non-invertible. It means that dynamics defined by (10) are underactuated on dynamic level.

\section{Concept of factitious force}

The mobile platform REX should be considered as an underactuated system because it has got a rectangular input matrix (which is non-invertible). There are two ways to solve the problem of inverting $B^{*}$ matrix (standing before control signals): 
- avoid a problem of missing control inputs assuming additional artificial constraints. Then a model in auxiliary velocities has the same size of reduced state variables as the number of control inputs,

- avoid a problem of missing control inputs assuming the additional factitious input. Then a model in auxiliary velocities has the same number of state variables and control inputs.

The first approach has been explored intensively in recent years. First attempt to solve the problem of underactuation in skid-steering mobile platforms can be found in [2], in which some additional assumption about lateral slipping has been done. Such an equation, although derived from the slipping effect, can be treated as a special nonholonomic constraint.

Another concept in control of the skid-steering platform can be found in [8]. The main idea of the proposed method was to add a control input to make $B^{*}$ square invertible matrix. Next, because this additional control does not exist in practice, it was assumed that it is equal to zero, i.e.

$$
u_{3 m} \equiv 0 \text {. }
$$

Such an approach can be used in other underactuated systems with greater deficit of control inputs.

3.1. Extension of input matrix $B^{*}$. Due to idea of factitious force, it is necessary to add one control input to the system (5). As a result of such an exertion, some extension of input matrix $B\left(q_{m}\right)$ defined in (8) has to be made

$$
B_{e}\left(q_{m}\right)=\left[B\left(q_{m}\right) \mid B_{3}\right]=\left[\begin{array}{cc|c}
0 & 0 & b_{1} \\
0 & 0 & b_{2} \\
0 & 0 & b_{3} \\
0 & 1 & b_{4} \\
1 & 0 & b_{5}
\end{array}\right] .
$$

The form of an input matrix (19) shows that it is possible to apply factitious force to any state variable of the platform REX.

From (11) we can calculate that input matrix for a model with factitious force can be expressed as

$$
B_{e}^{*}=G^{T} B_{e}=\left[\begin{array}{ccc}
\frac{2}{r} & 0 & h_{1} \\
0 & \frac{2}{r} & h_{2} \\
a_{5} & a_{4} & h_{3}
\end{array}\right]
$$

where elements $h_{i}$ are denoted as below

$$
\begin{gathered}
h_{1}=b_{1} \cos \theta+b_{2} \sin \theta+\frac{b_{3}}{c}+\frac{2}{r} b_{5}, \\
h_{2}=b_{1} \cos \theta+b_{2} \sin \theta-\frac{b_{3}}{c}+\frac{2}{r} b_{4}, \\
h_{3}=a_{1} b_{1}+a_{2} b_{2}+a_{3} b_{3}+a_{4} b_{4}+a_{5} b_{5} .
\end{gathered}
$$

Third condition. Matrix $B_{e}^{*}$ can be made invertible if only if

$$
\forall q_{m} \operatorname{det} B_{e}^{*} \neq 0,
$$

i.e.

$$
\forall q_{m}\left(\frac{2}{r} h_{3}-h_{1} a_{5}-h_{2} a_{4}\right) \neq 0 .
$$

3.2. Model in auxiliary velocities with additional input. Model of platform REX expressed in auxiliary velocities (10) with factitious force and column $G_{3}$ selected as in (12), can be presented as

$$
M^{*} \dot{\eta}+C^{*} \eta+F^{*}=B_{e}^{*} u_{m},
$$

with elements defined as follows

$$
\begin{aligned}
M^{*}= & {\left[\begin{array}{lll}
m_{11}^{*} & m_{12}^{*} & m_{13}^{*} \\
m_{12}^{*} & m_{11}^{*} & m_{23}^{*} \\
m_{13}^{*} & m_{23}^{*} & m_{33}^{*}
\end{array}\right], } \\
C^{*} & =\left[\begin{array}{ccc}
0 & 0 & C_{13}^{*} \\
0 & 0 & C_{23}^{*} \\
C_{31}^{*} & C_{32}^{*} & C_{33}^{*}
\end{array}\right], \\
F^{*} & =\left(\begin{array}{c}
-F_{x}-\frac{M_{r}}{c} \\
-F_{x}+\frac{M_{r}}{c} \\
-F_{y}
\end{array}\right),
\end{aligned}
$$

and input matrix $B_{e}^{*}$ given by (20).

Symbols occurring in model have meaning:

$$
\begin{gathered}
m_{11}^{*}=m_{t}+\frac{I_{p}}{c^{2}}+4 \frac{I_{x x}}{r^{2}}, \\
m_{12}^{*}=m_{t}-\frac{I_{p}}{c^{2}} \\
m_{13}^{*}=m_{t}\left(a_{1} \cos \theta+a_{2} \sin \theta\right)+\frac{a_{3} I_{p}}{c}+\frac{2}{r} a_{5} I_{x x}, \\
m_{23}^{*}=m_{t}\left(a_{1} \cos \theta+a_{2} \sin \theta\right)-\frac{a_{3} I_{p}}{c}+\frac{2}{r} a_{4} I_{x x}, \\
m_{33}^{*}=m_{t}\left(a_{1}^{2}+a_{2}^{2}\right)+I_{p} a_{3}^{2}+I_{x x}\left(a_{4}^{2}+a_{5}^{2}\right) .
\end{gathered}
$$

Elements of the Coriolis matrix, i.e. $C_{13}^{*}, C_{23}^{*}, C_{31}^{*}, C_{32}^{*}, C_{33}^{*}$ cannot be obtained explicitly because parameters $a_{i}$ can depend on control variables $q_{m}$. Therefore, they should be calculated only as Christoffel's symbols.

\section{Model of platform REX with $5 R$ arm}

The object considered in this paper is built with the holonomic rigid manipulator and nonholonomic differentially driven mobile platform REX. Mathematical model of nonholonomic system consists of two groups of equations: kinematics and dynamics. In this case kinematics denote nonholonomic constraints present in a mobile platform and dynamics have to be obtained for whole system including a platform and an onboard robotic arm.

The state vector of a mobile manipulator has a form

$$
\begin{aligned}
& q=\left(\begin{array}{ll}
q_{m} & q_{r}
\end{array}\right)^{T} \\
& =\left(\begin{array}{llllllllll}
x & y & \theta & \phi_{1} & \phi_{2} & q_{1} & q_{2} & q_{3} & q_{4} & q_{5}
\end{array}\right)^{T} \in R^{10}
\end{aligned}
$$


where $q_{m}$ are state variables associated with a platform and $q_{r}$ are manipulator's state variables.

In Fig. 1 differentially driven wheeled mobile platform REX was presented. A chassis has been modeled as homogeneous box with mass $m_{p}$ and moment of inertia $I_{z}$, equipped with four wheels with masses $m_{k}$ and moments of inertia $I_{z z}, I_{x x}$.

In turn, 5R manipulator presented in Fig. 2 was modeled as a set of three links, each treated as homogeneous stick of length $l_{i}$ and mass $m_{i}, i=1,2,3$. The last link with parameters $l_{4}$ and $m_{4}$, which is in fact gripper holding camera, was modeled as cuboid with $l, w$ and $h$ parameters.

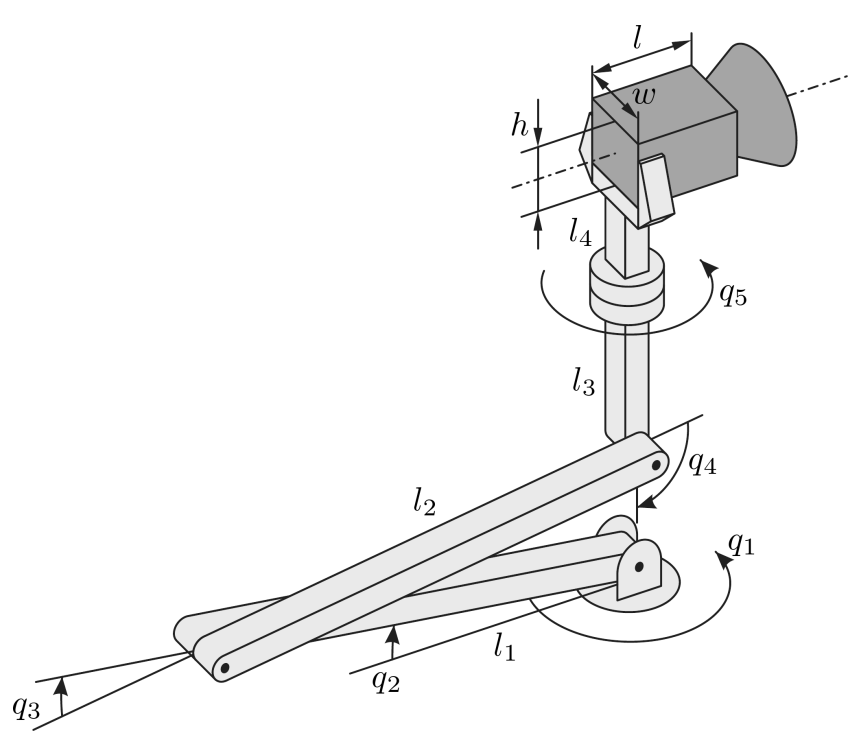

Fig. 2. Robotic arm $-5 R$ onboard manipulator

4.1. Dynamics of mobile manipulator. Special kind of wheeled mobile platforms are platforms with tracks. They can be modeled as a chassis with more than one axis equipped with fixed wheels, see Fig. 3. These platforms are called skidsteering mobile platforms, due to skidding effect observed in theirs behavior.

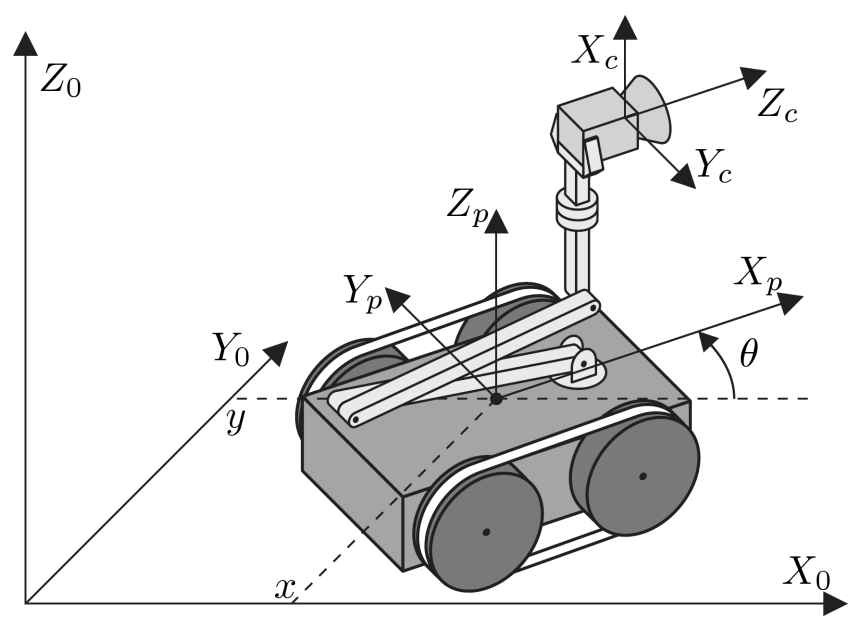

Fig. 3. Scheme of mobile manipulator with skid-steering platform REX
During the modeling process it has been assumed that a platform moves on an equipotential surface, therefore its potential energy equals to zero. It implies potential energy of a mobile manipulator depending only on the position of manipulating $5 \mathrm{R}$ arm. In turn, kinetic energy of a mobile manipulator is a sum of kinetic energy of a platform and of an onboard manipulator

$$
L=L_{p}+L_{m}=E_{k p}+E_{k m}-E_{p m} .
$$

Dynamics in generalized coordinates obtained from (22) have a block form

$$
\begin{gathered}
{\left[\begin{array}{cc}
M_{11} & M_{12} \\
M_{21} & M_{22}
\end{array}\right]\left(\begin{array}{c}
\ddot{q}_{m} \\
\ddot{q}_{r}
\end{array}\right)+\left[\begin{array}{cc}
M_{p} & 0 \\
0 & 0
\end{array}\right]\left(\begin{array}{c}
\ddot{q}_{m} \\
\ddot{q}_{r}
\end{array}\right)+\left(\begin{array}{c}
F \\
0
\end{array}\right)} \\
+\left[\begin{array}{ll}
C_{11} & C_{12} \\
C_{21} & C_{22}
\end{array}\right]\left(\begin{array}{c}
\dot{q}_{m} \\
\dot{q}_{r}
\end{array}\right)+\left(\begin{array}{c}
0 \\
D
\end{array}\right)=\left(\begin{array}{c}
B_{e} u_{m} \\
u_{r}
\end{array}\right)
\end{gathered}
$$

The detailed form of above matrix elements has been omitted in the text for sake of briefness.

If we extend dynamics (5) expressed in auxiliary velocities for nonholonomic skid-steering platform to the system composed on a platform and a manipulating arm (23), then we obtain the following equations

$$
M_{c}^{*}\left(\begin{array}{c}
\dot{\eta} \\
\ddot{q}_{r}
\end{array}\right)+C_{c}^{*}\left(\begin{array}{c}
\eta \\
\dot{q}_{r}
\end{array}\right)+D_{c}^{*}+F_{c}^{*}=B_{c}^{*}\left(\begin{array}{c}
u_{m} \\
u_{r}
\end{array}\right),
$$

where

$$
\begin{aligned}
& M_{c}^{*}=\left[\begin{array}{cc}
G^{T}\left(M_{11}+M_{p}\right) G & G^{T} M_{12} \\
M_{21} G & M_{22}
\end{array}\right], \\
& C_{c}^{*}=\left[\begin{array}{cc}
G^{T}\left(C_{11} G+\left(M_{11}+M_{p}\right) \dot{G}\right) & G^{T} C_{12} \\
M_{21} \dot{G}+C_{21} G & C_{22}
\end{array}\right], \\
& D_{c}^{*}=\left(\begin{array}{c}
0 \\
D
\end{array}\right) \\
& F_{c}^{*}=\left(\begin{array}{c}
G^{T} F \\
0
\end{array}\right)=\left(\begin{array}{c}
F^{*} \\
0
\end{array}\right) \text {, } \\
& B_{c}^{*}=\left[\begin{array}{cc}
G^{T} B_{e} & 0 \\
0 & I
\end{array}\right]=\left[\begin{array}{cc}
B_{e}^{*} & 0 \\
0 & I
\end{array}\right] .
\end{aligned}
$$

Equations (9) and (24) constitute a complete model of a nonholonomic mobile manipulator with the skid-steering platform, expressed in auxiliary coordinates. This model is a point of departure to design a control algorithm based on factitious force approach.

It is worth to mention that a mobile manipulator with a wheeled platform has a special property, which is not valid for its both subsystems, [13].

Property 1. For a mobile manipulator with a wheeled nonholonomic mobile platform a skew-symmetry between inertia matrix $M_{c}^{*}$ and the matrix of Coriolis and centripetal forces 
$C_{c}^{*}$ does not hold anymore. To regain the skew-symmetry, a special nontrivial correction matrix $C_{K}$ has to be added

$$
\frac{d}{d t} M_{c}^{*}=\left(C_{c}^{*}+C_{K}\right)+\left(C_{c}^{*}+C_{K}\right)^{T} .
$$

Any matrix, for which the relation (25) holds, can play a role of the correction matrix. The following expression describing a form of $C_{K}$ matrix, e.g.

$$
C_{K}=C_{K}^{T}=\frac{1}{2}\left\{M_{c}^{*}-C_{c}^{*}-\left(C_{c}^{*}\right)^{T}\right\}
$$

should be calculated before starting the regulation process.

\section{Control problem statement}

In this paper a mobile manipulator with the skid-steering mobile platform is considered. Such a robotic object should move along the desired trajectory without longitudinal slippage of its wheels. The desired trajectory is defined separately for each subsystem, i.e. a platform should move along the trajectory $q_{m d}(t)$ and the rigid onboard manipulator have to track a vector of the desired trajectory of joint positions $q_{r d}(t)$, defined relatively to the local frame of the platform.

Our goal is to address the following control problem:

1. Determine the control law $u$ such that mobile manipulator with the skid-steering platform with known dynamics follows the desired trajectory even if terrain parameters are unknown or some measurement disturbances occur.

2. During motion nonholonomic constraints have to be fulfilled - a platform should move without longitudinal slippage of its wheels.

3. All factitious forces have to be equal to zero, because they do not exist in reality.

In order to design a trajectory of the tracking controller for the considered object, it is necessary to consider a complete mathematical model of the nonholonomic system (9)-(24) expressed in auxiliary variables as a cascade composed of two groups of equations: kinematics (nonholonomic constraints) and dynamics, see Fig. 4:
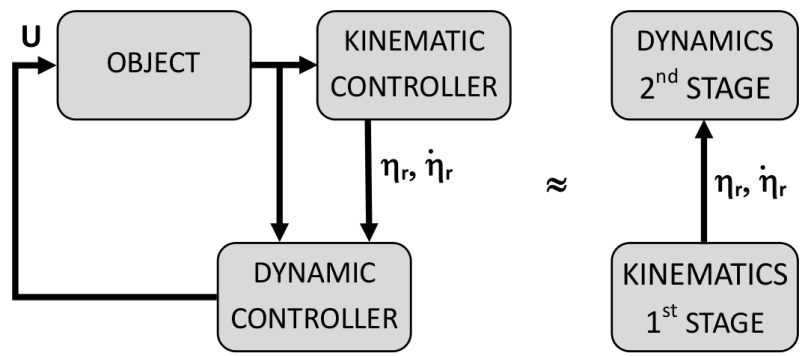

Fig. 4. Structure of the control algorithm (backstepping): cascade with two stages

For this reason the structure of the controller is divided into two parts working simultaneously (backstepping control approach, see [14]):

- kinematic controller $\eta_{r}$ - represents a vector of embedded control inputs, which ensure realization of the task for the kinematics (nonholonomic constraints) if the dynamics were not present. Such a controller generates 'velocity profile' which has to be executed in practice to realize the trajectory tracking for nonholonomic subsystem. Kinematic controller plays a role of 'motion planner'.

- dynamic controller - as a consequence of cascaded structure of the system model, the system's velocities cannot be commanded directly, as it has been assumed in the designing of kinematic control signals, and instead they must be realized as the output of the dynamics driven by $u$. Dynamics are calculated for the whole system, not for each subsystem separately.

\section{Main result - control law for mobile manipulator}

As we have mentioned in the previous section, the control algorithm consists of two parts: kinematic controller and dynamic controller. Both control algorithms, working simultaneously, are necessary to solve the control problem of a nonholonomic mobile manipulator.

6.1. Kinematic control algorithm. By designing the kinematic controller it is worth to notice, that matrix $G$ in (12) without an additional input, i.e. only with $G_{1}$ and $G_{2}$ columns, has the same form as the relevant matrix for unicycle. For this reason we have used the kinematic control law appropriate for a driving platform with only one fixed wheels axis. Such a control law is characterized by considerable robustness even noise and measurement errors occur during the regulation process [15]. In other words, we treat a signal coming from 'motion planner' as some approximation of skid-steering mobile platform motion.

Hence, the Samson controller developed for the unicycle can be used

$$
\begin{gathered}
v_{d}=\eta_{1 d}+\eta_{2 d}, \quad \omega_{d}=\frac{1}{c}\left(\eta_{2 d}-\eta_{1 d}\right), \\
\left(\begin{array}{c}
x_{e} \\
y_{e} \\
\theta_{e}
\end{array}\right)=\operatorname{Rot}(z,-\theta)\left(\begin{array}{c}
x_{d}-x \\
y_{d}-y \\
\theta_{d}-\theta
\end{array}\right), \\
\left(\begin{array}{c}
v_{r} \\
\omega_{r}
\end{array}\right)=\left(\begin{array}{c}
k_{1} x_{e}+v_{d} \cos \theta_{e} \\
\omega_{d}+k_{2} \theta_{e}+v_{d} y_{e} \frac{\sin _{\theta_{e}}}{\theta_{e}}
\end{array}\right), \quad k_{1}, k_{2}>0, \\
\eta_{1 r}=\frac{v_{r}-c \omega_{r}}{2}, \quad \eta_{2 r}=\frac{v_{r}+c \omega_{r}}{2},
\end{gathered}
$$

where $\eta_{1 d}, \eta_{2 d}$ and $x_{d}, y_{d}, \theta_{d}$ are desired velocities and position coordinates for an unicycle robot tracking the given trajectory; in turn, $\eta_{1 r}$ and $\eta_{2 r}$ are reference velocities signals, developed in a kinematic controller and used in the dynamic controller.

6.2. Dynamic control algorithm. Let's choose the dynamic control algorithm based on modification of the passivity-based sliding mode control law [16] given by Slotine \& Li for robotic manipulators. If we assume that dynamics of the mobile 
Implementation of factitious force method for control of $5 R$ manipulator with skid-steering platform REX

manipulator are known then we can propose the following control law

$$
\begin{gathered}
\left(\begin{array}{c}
u_{m} \\
u_{r}
\end{array}\right)=\left(B_{c}^{*}\right)^{-1}\left\{M_{c}^{*}\left(\begin{array}{c}
\dot{\eta}_{r} \\
\ddot{q}_{r e f}
\end{array}\right)+C_{c}^{*}\left(\begin{array}{c}
\eta_{r} \\
\dot{q}_{r e f}
\end{array}\right)\right. \\
\left.+D_{c}^{*}+F_{c}^{*}-C_{K}\left(\begin{array}{c}
e_{\eta} \\
s
\end{array}\right)-K_{d}\left(\begin{array}{c}
e_{\eta} \\
s
\end{array}\right)\right\},
\end{gathered}
$$

where $K_{d}=\operatorname{diag}\left\{k_{d}\right\}$ is a positive definite diagonal matrix and $C_{K}$ is the correction matrix necessary to get the skewsymmetry property (25).

Relevant elements can be defined as follows

$$
\begin{gathered}
e_{\eta}=\left(\begin{array}{c}
\eta_{1}-\eta_{1 r} \\
\eta_{2}-\eta_{2 r} \\
\eta_{3}-\eta_{3 r}
\end{array}\right), \\
s=\dot{q}_{r}-\dot{q}_{r e f}=\dot{e}_{q}+\Lambda e_{q}, \\
\Lambda=\Lambda^{T}>0, \\
e_{q}=q_{r}-q_{r d} .
\end{gathered}
$$

The closed-loop system (24) with feedback control (26) is given by

$$
M_{c}^{*}\left(\begin{array}{c}
\dot{e}_{\eta} \\
\dot{s}
\end{array}\right)+\left(C_{c}^{*}+C_{K}\right)\left(\begin{array}{c}
e_{\eta} \\
s
\end{array}\right)+K_{d}\left(\begin{array}{c}
e_{\eta} \\
s
\end{array}\right)=0 .
$$

6.3. Proof of convergence. For the system (28) we propose the following Lyapunov-like function

$$
V\left(e_{\eta}, s\right)=\frac{1}{2}\left(\begin{array}{ll}
e_{\eta} & s
\end{array}\right) M_{c}^{*}\left(\begin{array}{c}
e_{\eta} \\
s
\end{array}\right) \geq 0,
$$

which is non-negative definite. We compute time derivative of $V$ along solutions of the closed-loop system (28)

$$
\begin{gathered}
\dot{V}=\frac{1}{2}\left(\begin{array}{cc}
e_{\eta} & s
\end{array}\right) \dot{M}_{c}^{*}\left(\begin{array}{c}
e_{\eta} \\
s
\end{array}\right)+\left(\begin{array}{c}
e_{\eta} s
\end{array}\right) M_{c}^{*}\left(\begin{array}{c}
\dot{e}_{\eta} \\
\dot{s}
\end{array}\right) \\
=-\left(\begin{array}{cc}
e_{\eta} & s
\end{array}\right) K_{d}\left(\begin{array}{c}
e_{\eta} \\
s
\end{array}\right) .
\end{gathered}
$$

From La Salle \& Yoshizawa theorem, see [14] for details, it could be concluded that the errors $e_{\eta}$ and $s$ converge asymptotically to zero. Using definition of $s$ given by (27) and positive definiteness of parameter $\Lambda$ we get that position tracking error $e_{q}$ for manipulator joints goes asymptotically to zero.

On the other hand, the convergence of $e_{\eta}$ to zero means that the the velocity profile generated by a kinematic controller is successfully followed, and therefore one can conclude that the nonholonomic system i.e. skid-steering platform tracks the desired trajectory $q_{m d}$. It ends the proof.

\section{Simulation study}

For simulations we have applied the factitious force method with following parameters: third column of $G$ matrix and third column of $B$ matrix are equal to

$$
\left(\begin{array}{l}
a_{1} \\
a_{2} \\
a_{3} \\
a_{4} \\
a_{5}
\end{array}\right)=\left(\begin{array}{c}
-\sin \theta \\
\cos \theta \\
0 \\
0 \\
0
\end{array}\right) \quad\left(\begin{array}{l}
b_{1} \\
b_{2} \\
b_{3} \\
b_{4} \\
b_{5}
\end{array}\right)=\left(\begin{array}{c}
\sin \theta \\
-\cos \theta \\
0 \\
0 \\
0
\end{array}\right) .
$$

Such choice of parameters means that conditions (13), (16) and (22) are fulfilled.

7.1. Calculation of artificial signal $\eta_{3 r}$. The vector of auxiliary velocities consists of two groups of states: physical and factitious. Velocities $\eta_{1 r}$ and $\eta_{2 r}$ have physical meaning of the desired angular velocities of wheels on the left and right side of platform moving on the desired trajectory. In turn, third velocity $\eta_{3 r}$ is an auxiliary signal which can be calculated from the assumption that factitious force equals to zero. A form of such a signal strictly depends on the selected dynamic control algorithm.

The dynamic control law given by (26) implies the following form of factitious force

$$
\begin{gathered}
u_{3 m}=M_{c 11}^{*} \dot{\eta}_{1 r}+M_{c 12}^{*} \dot{\eta}_{2 r}+M_{c 13}^{*} \dot{\eta}_{3 r} \\
+M_{c 14}^{*} \ddot{q}_{1 r e f}+M_{c 15}^{*} \ddot{q}_{2 r e f}+M_{c 16}^{*} \ddot{q}_{3 r e f} \\
+M_{c 17}^{*} \ddot{q}_{4 r e f}+M_{c 18}^{*} \ddot{q}_{5 r e f}+C_{c 11}^{*} \eta_{1 r} \\
+C_{c 12}^{*} \eta_{2 r}+C_{c 14}^{*} \dot{q}_{1 r e f}+C_{c 15}^{*} \dot{q}_{2 r e f} \\
+C_{c 16}^{*} \dot{q}_{3 r e f}+C_{c 17}^{*} \dot{q}_{4 r e f}+C_{c 18}^{*} \dot{q}_{5 r e f} \\
+F_{c 3}^{*}-K_{d}\left(\eta_{3}-\eta_{3 r}\right) \equiv 0 .
\end{gathered}
$$

From (30) $\dot{\eta}_{3 r}$ can be derived as an implicit function $\left(C_{c 13}^{*}=\right.$ 0 ) and, in order to get $\eta_{3 r}$, it must be integrated - obtaining analytic form of the third reference signal is not possible. $\eta_{3 r}$ computed in this way fulfils the third subtask of a control problem.

7.2. Parameters. The simulations were run with MATLAB package and SIMULINK toolbox. As an object of simulations we have taken of the skid-steering mobile platform equipped with two axes of fixed wheels and 5R rigid manipulator. The parameters of the platform were: mass of the platform $m_{p}$, mass of the wheel $m_{k}$, platform moment of inertia $I_{z}$ relative $Z_{p}$ axis, wheel moment of inertia relative $Z_{p}$ axis $I_{z z}=\frac{1}{2} m_{k} r^{2}=2 I_{x x}$, half of platform width $c$, distances $a$ and $b$ from mass center to front and back axis of wheels.

Table 1

Simulation parameters

\begin{tabular}{lll}
\hline \hline$m_{p}=42 \mathrm{~kg}$ & $m_{k}=2.38 \mathrm{~kg}$ & $I_{z}=5 \mathrm{~kg} \cdot \mathrm{m}^{2}$ \\
\hline$I_{z z}=0.15 \mathrm{~kg} \cdot \mathrm{m}^{2}$ & $r=0.127 \mathrm{~m}$ & $m_{1}=3 \mathrm{~kg}$ \\
\hline$m_{2}=2 \mathrm{~kg}$ & $m_{3}=1 \mathrm{~kg}$ & $m_{4}=1 \mathrm{~kg}$ \\
\hline$l_{1}=1 \mathrm{~m}$ & $l_{2}=1 \mathrm{~m}$ & $l_{3}=l_{4}=0.5 \mathrm{~m}$ \\
\hline$l=0.3 \mathrm{~m}$ & $w=0.3 \mathrm{~m}$ & $h=0.5 \mathrm{~m}$ \\
\hline$a=0.365 \mathrm{~m}$ & $b=0.365 \mathrm{~m}$ & $c=0.387 \mathrm{~m}$ \\
\hline
\end{tabular}


A. Mazur and M. Cholewiński

In this section we want to show a behavior of the skidsteering mobile platform tracking different trajectories - admissible and inadmissible (obtained as bonding of different admissible trajectories). Simulations should show if the proposed control strategy works properly for mobile 5R manipulator with a skid-steering platform.

Desired joint trajectories have to track trajectories defined below

$$
q_{r d}=\left(\begin{array}{c}
q_{1 d}=3 \sin \left(\frac{t}{2}\right) \mathrm{rad} \\
q_{2 d}=0.5 \mathrm{rad} \\
q_{3 d}=0.1 \mathrm{rad} \\
q_{4 d}=0.1 \mathrm{rad} \\
q_{5 d}=0.3 \mathrm{rad}
\end{array}\right) .
$$

Regulation parameters have been chosen as follows: for dynamic controller $k_{d}=300$ and $\Lambda=1$, for kinematic controller $k_{1}=1$ and $k_{2}=1$.

7.3. Admissible trajectory. The desired admissible trajectory has been chosen as a circle with radius $R_{t r a j}=10 \mathrm{~m}$ and frequency $\omega_{d}=0.1 \frac{\mathrm{rad}}{\mathrm{s}}$. From the control point of view such a trajectory is most exacting because a mobile platform has persistent lateral slippage during motion.

A real trajectory realized by the platform can be observed in Fig. 5. Tracking errors of manipulator joints have been presented in Fig. 6.

7.4. Inadmissible trajectory. The desired inadmissible trajectory has been chosen as a square trajectory. From the control point of view such a trajectory is interesting because some problems with bonding points occur.

A real trajectory realized by the platform can be observed in Fig. 7. Tracking errors of manipulator joints have been presented in Fig. 8.

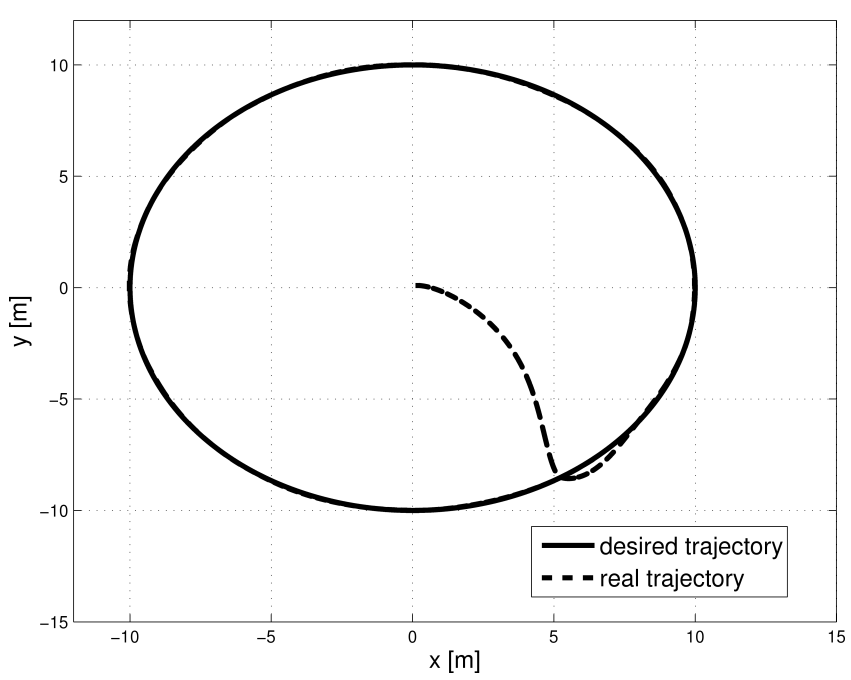

Fig. 5. Admissible trajectory realized by skid-steering platform

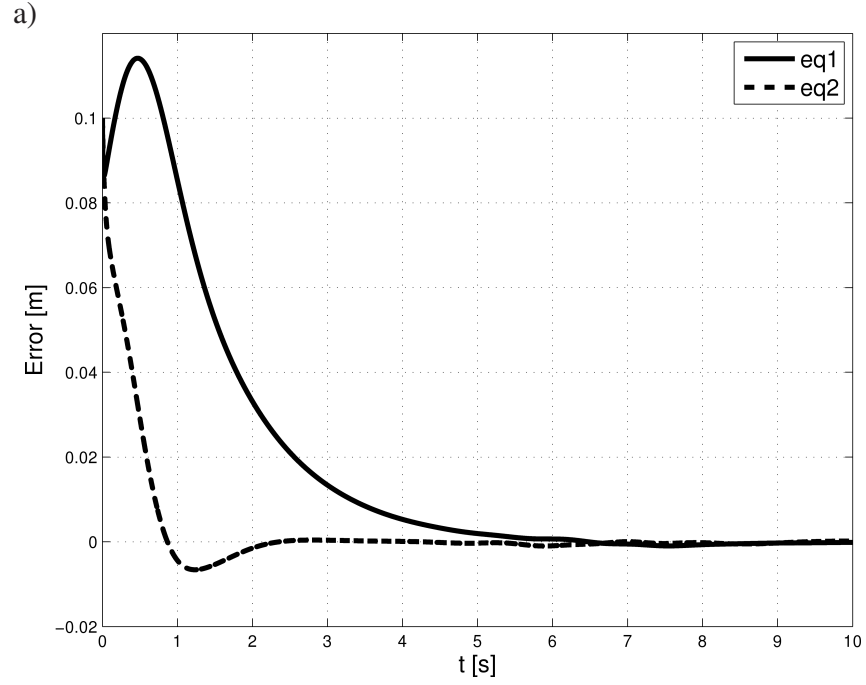

b)

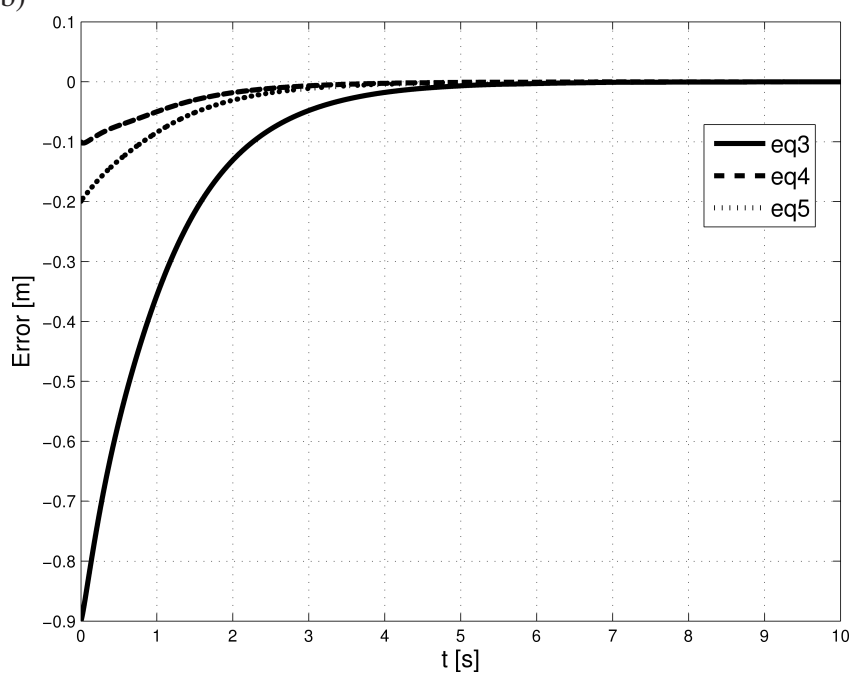

Fig. 6. Errors obtained by trajectory tracking: a) joint errors $e_{q 1}, e_{q 2}$ of manipulator, b) joint errors $e_{q 3}, e_{q 4}$ and $e_{q 5}$ of manipulator

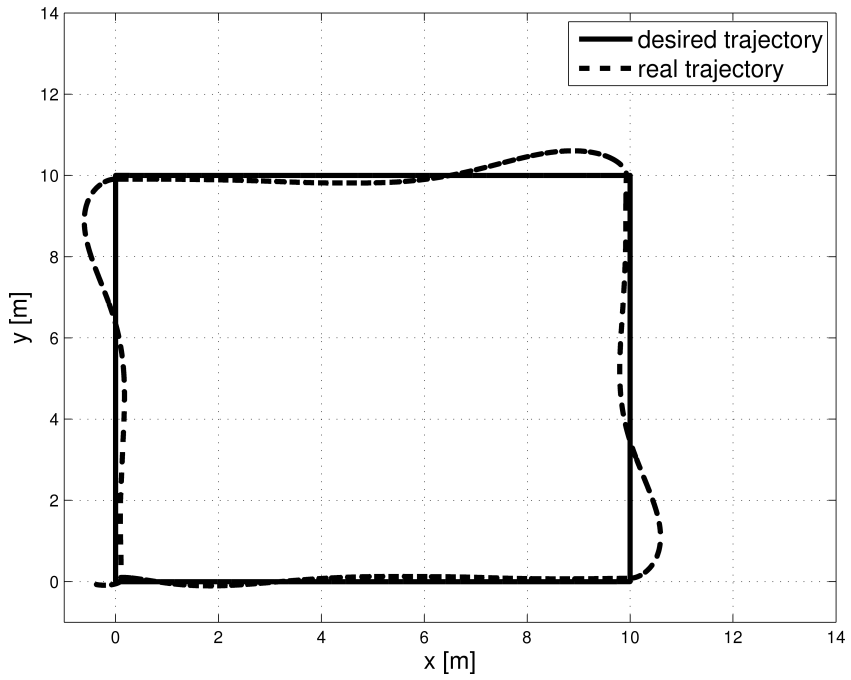

Fig. 7. Inadmissible trajectory realized by skid-steering platform 
a)

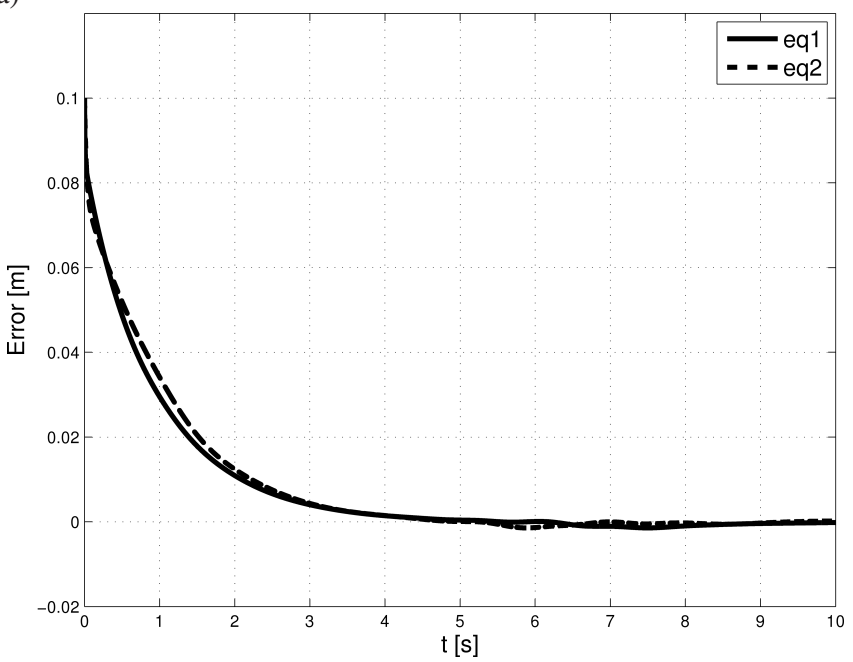

b)

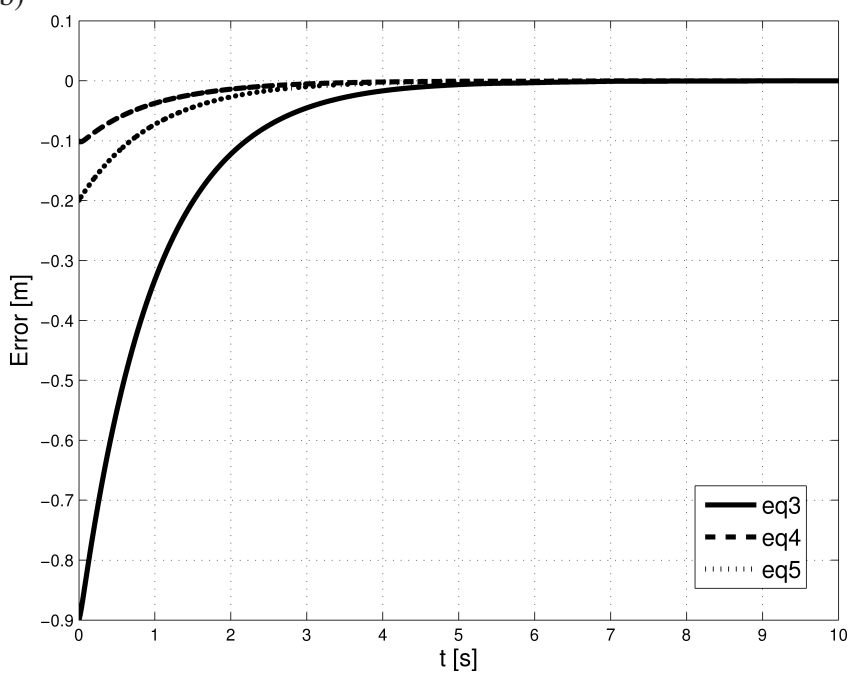

Fig. 8. Errors obtained by trajectory tracking: a) joint errors $e_{q 1}, e_{q 2}$ of manipulator, b) joint errors $e_{q 3}, e_{q 4}$ and $e_{q 5}$ of manipulator

It can be observed that after 10, 20, 30 and $40 \mathrm{~s}$ the desired trajectory changes from one side of the square to the next one. It means that for these time moments trajectory has to change a signal coming from kinematic control from one solution (obtained for one side of the square) to the another (obtained for the next side). In other words, in these time moments some impulse in tracking errors should occur.

\section{Conclusions}

In the paper the idea of control mobile manipulator with a skid-steering mobile platform has been presented. It has been shown that a nonholonomic system with velocity constraints has a cascaded structure, which requires two simultaneously working controllers: kinematic controller ('motion planner') and dynamic controller. The most crucial fact is too small number of dynamic control inputs relatively to a number of state variables - it means that the system is underactuated on a dynamic level. In this article an attempt to solve such a problem with so-called "factitious force" has been made. The concept of factitious force assumes that dynamics have additional control inputs $u_{v}$, which do not exist in reality and are equal to zero. However, such an assumption makes possible to invert the input matrix, standing before control inputs in dynamic equations.

The factitious force method and the proposed control algorithms have been examined in simulations for a mathematical model of a mobile manipulator with $5 \mathrm{R}$ degrees of freedom and the skid-steering mobile platform REX, built under the grant RobREx. By theoretical considerations and simulation study it was confirmed that the control method introduced in the paper works properly. The examined control algorithm is implemented in the real REX platform.

In future works the factitious force concept will be extended to control other dynamically underactuated nonholonomic systems and to the control skid-steering wheeled mobile platform with other nonholonomic constraints coming e.g. from lack of lateral or longitudinal slippage only selected axes or wheels.

Acknowledgements. This work was supported by the National Centre for Research and Development under the grant RobREx PBS1/A3/8/2012, PS0065.

\section{REFERENCES}

[1] A. Mazur, "New approach to designing input-output decoupling controllers for mobile manipulators", Bull. Pol. Ac.: Tech. 53 (1), 31-37 (2005).

[2] L. Caracciolo, A. De Luca, and S. Iannitti, "Trajectory tracking control of a four-wheel differentially driven mobile robot", Proc. IEEE Int. Conf. on Robotics and Automation 4, 26322638 (1999).

[3] K. Kozłowski and D. Pazderski, "Modeling and control of a 4wheel skid-steering mobile robot", Int. J. Appl. Math. Comput. Sci. 14 (4), 477-496 (2004).

[4] A. Mazur and M. Cholewiński, "Robust control of differentially driven mobile platforms", Proc. 8th Workshop Robot Motion and Control RoMoCo 2011 1, 53-64 (2011).

[5] D. Pazderski and K. Kozłowski, "Trajectory tracking of underactuated skid-steering robot", Proc. American Control Conf. 4, 3506-3510 (2008).

[6] E. Mohammadpour, M. Naraghi, and M. Gudarzi, "Posture stabilization of skid steer wheeled mobile robots", Proc. IEEE Int. Conf. on Robotics, Automation and Mechatronics 1, 163-169 (2010).

[7] E. Maalouf, M. Saad, and H. Saliah, "A higher level path tracking controller for a four-wheel differentially steered mobile robot", Robotics and Autonomous Systems 1, 23-33 (2006).

[8] A. Mazur and M. Cholewiński, "Virtual force concept in steering mobile manipulators with skid-steering platform moving in unknown environment", J. Intell. Robot. Syst. 77, 433-443 (2015).

[9] I. Motte and G. Campion, "A slow manifold approach for the control of mobile robots not satisfying the kinematic constraints", IEEE Trans. Rob. Autom. 16 (6), 875-880 (2000).

[10] K. Tchoń, A. Mazur, I. Dulȩba, R. Hossa, and R. Muszyński, Manipulators and Mobile Robots: Models, Motion Planning, Control, PLJ Publisher, Warsaw, 2000, (in Polish). 
A. Mazur and M. Cholewiński

[11] I. Dulęba, Methods and Motion Planning Algorithms of Mobile and Manipulating Robots, Academic Printing House EXIT, Warsaw, 2001.

[12] C. Canudas de Wit, B. Siciliano, and G. Bastin, Theory of Robot Control, Springer, London, 1996.

[13] I. Dulęba, "Modeling and control of mobile manipulators", Proc. 6th IFAC Symp. Robot Control 2000 SYROCO'00 1, 687-692 (2000).
[14] M. Krstić, I. Kanellakopoulos, and P. Kokotović, Nonlinear and Adaptive Control Design, J. Wiley, New York, 1995.

[15] U. Libal and J. Płaskonka, "Noise sensitivity of selected kinematic path following controllers for a unicycle", Bull. Pol. Ac.: Tech. 62 (1), 3-13 (2014).

[16] J.J. Slotine and W. Li, "Adaptive manipulator control: a case study", IEEE Trans. Autom. Contr. 33 (11), 995-1003 (1988). 